\title{
Object-recognition memory is only mildly impaired in rats with lesions of the hippocampus and amygdala
}

\author{
DAVE G. MUMBY, EMMA R. WOOD, and JOHN P. J. PINEL \\ University of British Columbia, Vancouver, British Columbia, Canada
}

\begin{abstract}
Rats were trained on the nonspatial delayed nonmatching-to-sample task that we recently developed to mimic the task that is commonly used to study amnesia in monkeys. The rats were tested at retention delays of 4, 15, 60, 120, and $600 \mathrm{sec}$, both before and after surgery. Each experimental rat received bilateral lesions of the hippocampus, amygdala, or both. In comparison to no-surgery control rats, the rats in each of the three experimental groups were significantly and equally impaired only at the $600-\mathrm{sec}$ delay. These findings indicate that neither separate nor combined lesions of the amygdala and hippocampus cause severe object-recognition impairments in pretrained rats.
\end{abstract}

Bilateral damage to the medial temporal lobes has been shown to produce severe anterograde amnesia in humans and monkeys. Although experiments using monkey models of medial-temporal-lobe amnesia have helped to elucidate the structures whose damage contributes to it, there is still controversy over this issue. The most widely used monkey model involves an object-recognition task, the nonrecurring-items delayed nonmatching-to-sample (DNMS) task, which has been shown to be sensitive to medial-temporal-lobe damage in humans (Squire, ZolaMorgan, \& Chen, 1988). Impairments on DNMS in monkeys have been found following damage to the hippocampus (Bachevalier \& Mishkin, 1989; Mahut, ZolaMorgan, \& Moss, 1982; Zola-Morgan \& Squire, 1985) or amygdala (Murray \& Mishkin, 1984). Other studies have found impairments on DNMS in monkeys only if both structures (e.g., Mishkin, 1978) or their primary efferents (Bachevalier, Parkinson, \& Mishkin, 1985) have been damaged. However, recent evidence suggests that impairments on DNMS in monkeys following lesions of the hippocampus and amygdala may result from incidental cortical damage (Murray \& Mishkin, 1986; ZolaMorgan, Squire, \& Amaral, 1989; Zola-Morgan, Squire, Amaral, \& Suzuki, 1989).

Recently, the controversy over the role of the hippocampus and the amygdala in recognition memory has extended to research on rats. Olton and Feustle (1981) found impaired DNMS performance in rats with hippocampal lesions, whereas Aggleton, Hunt, and Rawlins (1986) and

This research was supported by a grant to J.P.J.P. from the British Columbia Health Care Research Foundation. D.G.M. was supported by a postgraduate studentship from the Medical Research Council of Canada. We thank Eytan David, Tod Kippen, and Kate Banks for their assistance in collecting the data, and Betsy Murray for her many excellent comments on an earlier version of this manuscript. Correspondence should be addressed to Dave G. Mumby, Department of Psychology, University of British Columbia, Vancouver, BC, Canada V6T 1Z4.
Rothblat and Kromer (1991) did not. Aggleton, Blindt, and Rawlins (1989) found no impairments on DNMS following amygdalar lesions, but they observed dramatic impairments following combined amygdalo-hippocampal lesions; however, they emphasized that collateral damage to pyriform cortex may have contributed to the impairments displayed by the rats with amygdalo-hippocampal lesions.

There is evidence that the amount of presurgery training on the DNMS task may influence the magnitude of postsurgery deficits. For example, hippocampal lesions have a less disruptive effect on DNMS performance in monkeys that receive presurgery training (e.g., Mishkin, 1978; Murray \& Mishkin, 1984) than they do in monkeys that do not (e.g., Zola-Morgan \& Squire, 1986). Presurgery training has advantages and disadvantages. On one hand, postsurgery testing of subjects that have not had presurgery training is more likely to reveal an anterograde effect of a lesion. On the other, presurgery training is needed to detect retrograde memory deficits. Presurgery training also allows one to establish stable baselines of performance for individual subjects to which their postsurgery performance can be compared. More importantly, presurgery training eliminates postsurgery deficits that are caused by impaired acquisition of the nonmnemonic skills that are required for successful performance, thus making observed deficits easier to interpret.

In the present experiment, we assessed the effects of hippocampal, amygdalar, and combined amygdalohippocampal lesions on rats' performance of the DNMS task that we recently developed to mimic the DNMS task that has been used to model amnesia in brain-damaged monkeys (Mumby, Pinel, \& Wood, 1990). Our objective was to determine whether medial-temporal-lobe amnesia could be similarly modeled in rats. The rats received extensive training on the DNMS task prior to surgery. After postsurgery testing, some of the rats received an additional bilateral lesion so that they would have combined 
amygdalo-hippocampal lesions, and then they were retested. Thus, some of the rats with amygdalo-hippocampal lesions received one-stage lesions, and the others received two-stage lesions. We chose to make aspiration lesions of the hippocampus rather than neurotoxic lesions because the former method, although not as selective as the latter, enables more complete lesions of the hippocampal formation to be made. We felt that it was expedient to begin the study of our DNMS paradigm by assessing the effects of complete lesions. Furthermore, one of our major aims was to compare the effects of similar hippocampal lesions in rats and monkeys-hippocampal lesions have been made by aspiration in most of the comparable monkey studies.

\section{METHOD}

\section{Subjects}

The subjects were 28 experimentally naive, male Long-Evans rats (Charles River, Quebec) that were between 8 and 12 weeks old at the beginning of the experiment. They were housed individually with continuous access to water under a 12:12 h light:dark cycle, with light onset at 8:00 a.m. Their body weights were maintained at approximately $85 \%$ of ad-lib levels throughout the experiment by the administration of daily rations of rat chow. Training began after the rats had been on the restricted feeding regimen for 14 days.

\section{Apparatus}

The apparatus (see Figure 1) has been described in detail elsewhere (Mumby et al., 1990). Briefly, it consists of an elevated runway, which is separated from identical goal areas at each end by opaque guillotine doors. Each goal area contains two food wells into which food pellets (45 $\mathrm{mg}$ Bio-Serv Inc., Frenchtown, NJ) can be delivered by hand through plastic tubes that are mounted on the outside of the apparatus. The two food wells are separated by a short divider wall $(9 \times 9 \mathrm{~cm})$, which protrudes from the center of the end wall. The sides of the goal areas are open to allow the experimenter to easily place stimulus objects over the food wells and to remove them quickly.

We used a collection of over 350 test objects of various shapes, textures, and colors, similar to the "junk" objects that are used in the monkey DNMS paradigm (see Mishkin \& Appenzeller, 1987). Each object was large enough to cover a food well but small enough and light enough to be easily displaced by the rats. Some of these objects may have had distinctive odors, but no objects with obvious scents were included.

\section{Procedures}

The training and testing procedures have been previously described in detail (see Mumby et al., 1990). All testing occurred during the light phase of the light:dark cycle, between 14 and $21 \mathrm{~h}$ after the rat's most recent meal. Each rat was tested no more than once per day and no less than three times per week. The rats were not handled during a session once they had been placed in the apparatus. Each rat was habituated to the apparatus, and then it progressed through three phases of presurgery training and testing: (1) acquisition of a simple object-discrimination task, (2) acquisition of the DNMS task, and (3) determination of its DNMS retention function. Following recovery from surgery, each rat received two phases of postsurgery testing: (1) reacquisition of the DNMS task with a brief retention delay, and (2) determination of its postsurgery DNMS retention function.

Habituation. The habituation phase consisted of six daily 20-min sessions. In Sessions 1 and 2, each rat was placed in the apparatus

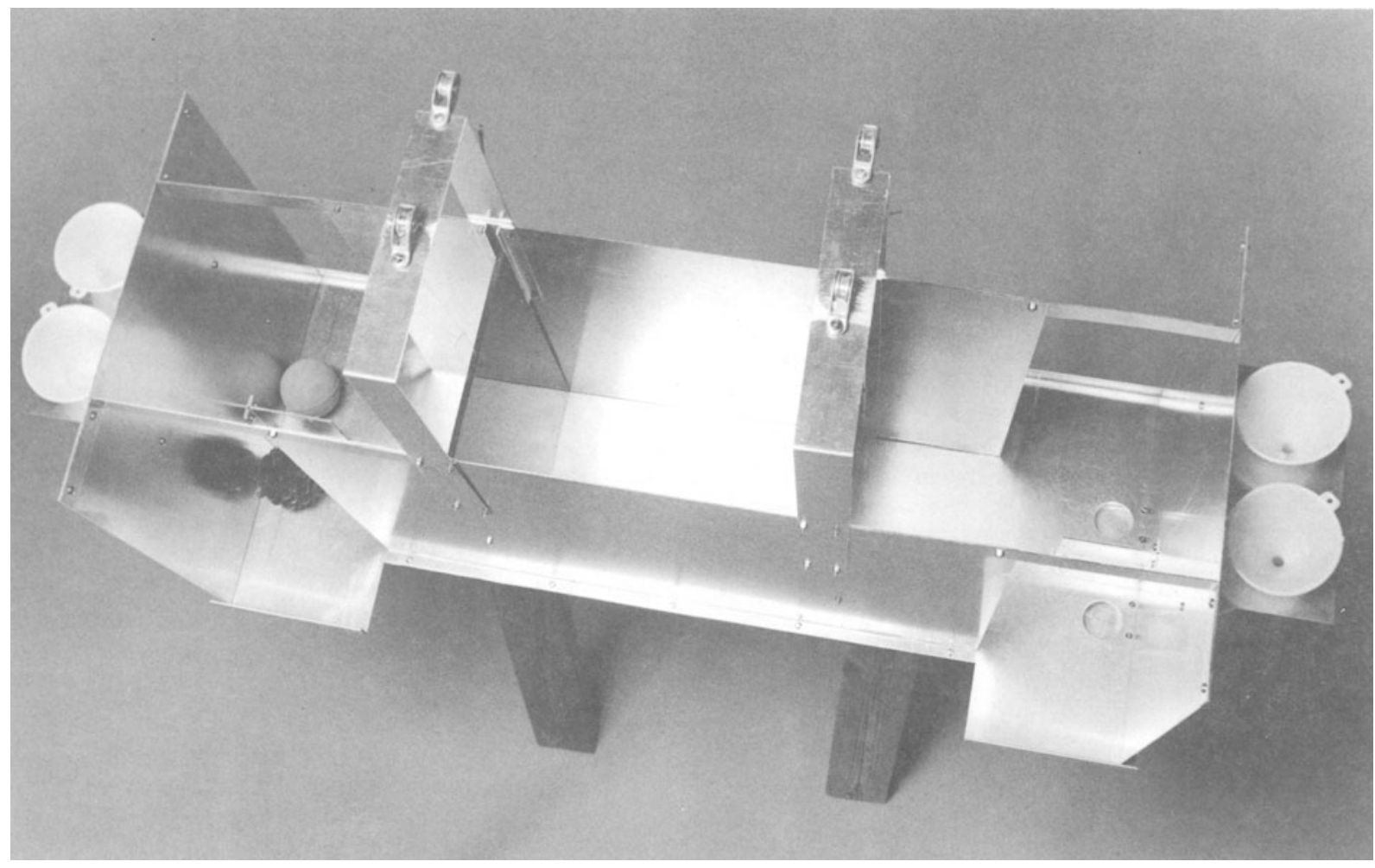

Figure 1. The nonrecurring-items delayed nonmatching-to-sample apparatus. (From Psychobiology, 18, 321-326, 1990; reprinted by permission.) 
and was allowed to explore and to find food pellets inside the food wells. In Sessions 3 and 4, the rats were shaped to run back and forth between the goal areas by alternately baiting a single well at each end. The guillotine doors remained open during Sessions 1-4. In Sessions 5 and 6, the guillotine doors were closed, and the experimenter shaped the rats to approach the doors by baiting a food well on the other side and raising the door when the rat approached it.

Presurgery training: Acquisition of object discrimination. Following the 6-day habituation phase, each rat received either four or five object-discrimination sessions, each comprising 25 trials. For each subject, the same two test objects served as the stimuli for all of its object-discrimination trials. One of the objects was designated S+ (reward); the other object was designated S- (no reward). To begin each session, the rat was placed in the center of the apparatus; one door was open, the other was closed. The $\mathrm{S}+$ and $\mathrm{S}-$ were each placed over the food wells behind the closed door-the position of $\mathbf{S}+$ (left or right) varied from trial to trial according to a pseudorandom pattern. Then, the experimenter opened the door to expose the two objects. When the rat approached and displaced an object, the far door was lowered. If the rat displaced S+, a food pellet was then delivered to that food well; if $S$ - was displaced, no food pellet was delivered. The experimenter then positioned $\mathrm{S}+$ and $\mathrm{S}-$ at the other end of the apparatus in preparation for the next trial. The duration of the intertrial interval varied, but it was typically between 15 and $20 \mathrm{sec}$. Two special procedures were employed on the first discrimination session but not thereafter: (1) To encourage the rats to displace the objects, the well under the S+ was prebaited so that they could smell the food, and (2) if the rats displaced $S-$, they were allowed to then displace $S+$ to obtain a reward before the experimenter removed the objects.

Presurgery training: Acquisition of DNMS. The 350 test objects were divided into seven sets of 50 . Different sets were used on successive sessions, and different pairs of objects were used for each of the trials within a session. Each rat received either 20 or 25 trials per session on all of its DNMS sessions. The number of trials per session had been set at 25 for the first several subjects, but it was reduced to $\mathbf{2 0}$ for the subsequent subjects so that more rats could be tested each day.

To begin each session, the rat was placed in the apparatus, and the doors were lowered to enclose it in the central starting area. Before each trial, a food pellet was placed in one of the food wells, the sample object was placed over it, and the novel object was placed over one of the food wells at the other end of the apparatus. The location of the sample and novel objects varied from trial to trial according to a pseudorandom pattern.

To begin a trial, the experimenter raised the door to allow access to the sample object, which the rat approached and displaced from the food well. While the rat ate the pellet, the experimenter removed the sample object and positioned it over the vacant food well at the other end of the apparatus. After the prescribed delay, the other door was raised and the rat approached and displaced either the sample object or the novel object. If it displaced the novel object, a food pellet was delivered to the exposed food well; if it displaced the sample object, no pellet was delivered. A rat was considered to have displaced an object only if it moved the object enough to expose the food well. Then, the experimenter removed the objects, the rat returned to the central starting area, and the doors were closed to confine the rat there until the next trial, which began after new sample and novel objects were positioned. The intertrial interval varied, but it was typically between 20 and $30 \mathrm{sec}$. The rats were permitted to make corrections during the first two DNMS sessions but not thereafter.

The delay was defined as the time between the displacement of the sample object by the rat and the raising of the door to reveal the sample and novel objects. The delay was approximately $4 \mathrm{sec}$ during the initial sessions. For each rat, training continued at this 4-sec delay until it reached a criterion of at least $84 \%$ of trials cor- rect on two consecutive sessions, whereupon the delay was increased to $15 \mathrm{sec}$. The delay was subsequently increased to $30,60,120$, and then finally to $600 \mathrm{sec}$ whenever a rat either reattained the criterion or had eight sessions at a particular delay without reaching the criterion. Four sessions were conducted at the 600 -sec delay.

Presurgery testing: Determination of retention functions. Each rat's retention function was assessed during the final phase of presurgery testing. The rats received either 5 or 10 mixed-delay sessions, each consisting of 25 trials. On these sessions, 5 trials were conducted at each of the following delays: $4,15,60,120$, and $600 \mathrm{sec}$. These delays appeared in the following order in each session: $4 \mathrm{sec}, 15 \mathrm{sec}, 60 \mathrm{sec}, 120 \mathrm{sec}, 600 \mathrm{sec}, 600 \mathrm{sec}, 120 \mathrm{sec}$, $60 \mathrm{sec}, 15 \mathrm{sec}, 4 \mathrm{sec}, 4 \mathrm{sec}, 15 \mathrm{sec}$, and so on. Although we had previously found that performance was stable over 5 mixed-delay sessions (see Mumby et al., 1990), we thought that it might improve if the rats received more testing. Accordingly, some rats in the present experiment were given 10 presurgery mixed-delay sessions.

Surgery. Following presurgery testing, each rat received bilateral aspiration lesions of the hippocampus $(n=11)$, electrolytic lesions of the amygdala $(n=7)$, combined amygdalo-hippocampal lesions $(n=4)$, or was assigned to a no-surgery control group $(n=6)$. We did not include a control group comprising rats with neocortical lesions similar to those that accompany hippocampal aspiration lesions because the results of pilot experiments indicated that such lesions have no obvious effects on the DNMS performance of pretrained rats; these pilot data are described at the end of the Results section. Surgery was performed under pentobarbital anesthesia (60 mg/kg).

In preparation for hippocampal aspiration, the scalp was incised, and holes were drilled on each side of the skull, each hole extending from approximately $2 \mathrm{~mm}$ posterior to the coronal suture to $2 \mathrm{~mm}$ anterior to the lamboid suture and from $1.5 \mathrm{~mm}$ lateral to the sagittal suture to within $1 \mathrm{~mm}$ of the temporal ridge. Then, the exposed dura mater was cut and the underlying neocortex and white matter were aspirated with a glass Pasteur pipette to expose the dorsal hippocampus. Next, the dorsal hippocampus and part of the lateral hippocampus were aspirated, and the cavity was filled with Gelfoam (Upjohn Co., Don Mills, Ontario). After the skin was sutured, the rats were placed under a heat lamp in a recovery room. Diazepam (10-15 $\mathrm{mg} / \mathrm{kg}$, i.p.) was administered as soon as they began to regain consciousness, and for the next $24 \mathrm{~h}$ smaller doses were periodically administered to control the convulsions that sometimes $o c$ cur in rats after hippocampal lesions are made. Few convulsions were observed in the present subjects. The rats were returned to their home cages on the day after surgery.

The amygdalar lesions were made bilaterally at three sites with a bipolar stainless steel wire electrode ( $2 \mathrm{~mA}$ for $20 \mathrm{sec}$ ), which was insulated with Teflon except for approximately $1 \mathrm{~mm}$ at its tip. The following were the electrode coordinates relative to bregma: AP -2.3, ML -4.5, DV -9.8; AP -3.3, ML - 4.5, DV - 10.0; AP $-4.3, \mathrm{ML}-4.5, \mathrm{DV}-10.0$. The rats were returned to their home cages immediately after amygdalar surgery.

The above procedures for hippocampal and amygdalar surgery were combined for the rats that received one-stage amygdalohippocampal lesions. These rats received the same postsurgical care as the rats that received restricted hippocampal lesions.

Before testing resumed, the experimental rats were allowed at least 14 days to recover from surgery, and the no-surgery control rats were not tested for 14 days. During the first 10 days, each rat had continuous access to food, after which they were returned to a restricted feeding regimen.

Postsurgery testing: Reacquisition of DNMS. Following recovery, the rats were tested on the DNMS task at 4-sec delays until they either reattained the original performance criterion or completed 20 sessions without attaining it.

Postsurgery testing: Determination of retention functions. Next, the rats were given mixed-delay sessions in which were used 
the same procedures that had been used to determine their presurgery retention functions. Each rat received the same number of mixeddelay sessions after surgery as it had received before surgery (i.e., either 5 or 10 ).

Some of the rats then received a second operation; 5 rats with amygdalar lesions received hippocampal lesions, and 7 rats with hippocampal lesions received amygdalar lesions. Following recovery, these rats were treated and tested as they had been following their first lesion.

\section{RESULTS}

Anatomical. Figure 2 shows the location and the extent of the largest and smallest amygdalo-hippocampal lesions. In several of the rats with two-stage amygdalo-hippocampal lesions, the amygdalar and hippocampal lesions overlapped to form a continuous lesion, thus making it impossible to determine the boundaries of the damage sustained during each surgery. Accordingly, Figure 2 also shows the damage sustained by 1 of the rats with hippocampal lesions that did not subsequently receive amygdalar lesions and by 1 of the rats with amygdalar lesions that did not subsequently receive hippocampal lesions; the damage in these 2 rats was representative of that sustained by the other rats with hippocampal and amygdalar lesions.

Most of the hippocampal lesions included the entire dorsal hippocampus, most of the lateral hippocampus, and a portion of the neocortex and corpus callosum overlying the dorsal hippocampus. The ventral extent of most of the hippocampal lesions spared small portions of the dentate gyrus and subiculum. The caudal extent of these lesions varied in the amount of presubiculum and parasubiculum that was removed. Each lesion extended rostrally to include the fimbria fornix, and two of them included slight unilateral damage to dorsal portions of the lateral septal nucleus. Some rats also sustained small unilateral lesions in the caudate nucleus.

Small infarcts were also present in the dorsal thalamus of some of the brains with hippocampal lesions. Most of these were unilateral and involved the habenular nuclei, lateral dorsal nucleus, lateral pulvinar nucleus, lateral geniculate, and medial geniculate nucleus; damage to these thalamic nuclei appeared to be unrelated to the behavioral results. Some of the brains with hippocampal lesions also received partial unilateral damage to the colliculi.

Four of the rats with hippocampal lesions sustained cortical damage that was much more extensive than in the other rats in that group (see Figure 5). This damage was unintended, and it included portions of entorhinal, perirhinal, and temporal association cortex (area Te2; Zilles, 1985). Accordingly, the behavioral data from these 4 rats were excluded from the overall statistical analyses, and they are dealt with separately at the end of the Results section. The extent of damage to the thalamus and colliculi in the 4 rats was not unlike that found in the other rats with hippocampal lesions; however, 1 of them sustained large bilateral lesions of the lateral septal nucleus.

The amygdalar lesions varied in the extent of damage to specific nuclei, but the most consistent damage was to the medial two-thirds of the amygdaloid complex. No specific amygdaloid nuclei were consistently spared. The caudal extent of some of the amygdalar lesions included small portions of medial entorhinal cortex (see the largest amygdalo-hippocampal lesion in Figure 2), but the presence of such damage did not appear to be related to the behavioral results.

Presurgery training: Acquisition of object discrimination. All rats quickly learned the object-discrimination task. On Session 2, the first session on which they were not allowed to correct their errors, scores averaged $68 \%$ (ranging from $44 \%$ to $88 \%$ ), and on the final session, scores averaged $92 \%$ (ranging from $76 \%$ to $100 \%$ ).

Presurgery training: Acquisition of DNMS. Rats required a mean of 280 trials $(S E=22.7)$ to reach the performance criterion at the 4-sec delay. There were no significant differences in the number of trials to criterion or in the presurgery percent-correct scores between rats that received 20 trials per session and those that received 25 trials per session, so the data from all of the rats were pooled for the purpose of statistical analysis. Most rats' scores dropped each time that the delay was lengthened and then recovered over subsequent sessions. Excluding the first two sessions at each delay, the mean-percentcorrect scores at each delay during acquisition training were almost identical to those obtained during the subsequent mixed-delay sessions.

Presurgery testing: Retention functions. As shown in Figure 3, scores on the presurgery mixed-delay sessions declined significantly as the delay was lengthened $[F(4,115)=52.6, p<.001]$. There were no significant differences among the groups' presurgery scores at any delay. One-sample $t$ tests revealed that the scores in each group were significantly better than chance at the 600 sec delay (all $p s<.05$; one-tailed). Performance at all delays was stable over the mixed-delay sessions regardless of whether the rats received 5 or 10 of these sessions.

Postsurgery testing: Reacquisition of DNMS. On the first few trials of the first postsurgery testing session, many of the lesioned rats were slow to approach the goal areas when the doors were raised, but most moved normally by the end of that session. Each rat readily displaced test objects from the food wells during the first postsurgery session and continued to do so thereafter.

An analysis of variance indicated that the postsurgery performance of rats with one-stage amygdalo-hippocampal lesions was similar to that of rats with two-stage amygdalohippocampal lesions on mixed-delay sessions $[F(1,14)<$ 1]. Therefore, the data from all 16 rats with amygdalohippocampal lesions (4 with one-stage lesions and 12 with two-stage lesions) were pooled for the purposes of statistical analysis and the presentation of results in Figure 3. The mean $(S E)$ number of postsurgery trials at the 4-sec delay that were required to reattain the criterion by the no-surgery controls, and by the rats with hippocampal, amygdalar, and amygdalo-hippocampal lesions, were 66.7 (66.7), $53.6(10.1), 40.7(19.8)$, and $66.2(22.4)$, respectively. These differences were not statistically significant. 
Amygdalo-hippocampal

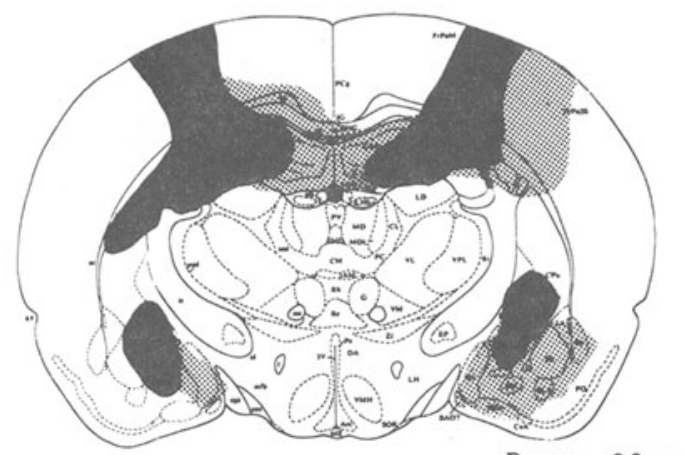

Bregma $-2.3 \mathrm{~mm}$

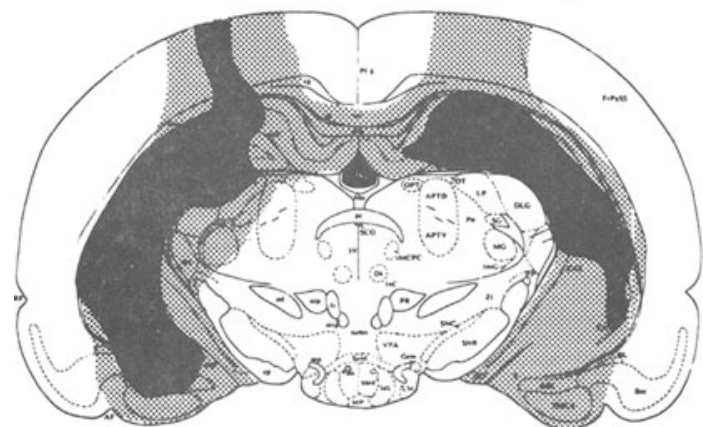

Bregma $-4.8 \mathrm{~mm}$

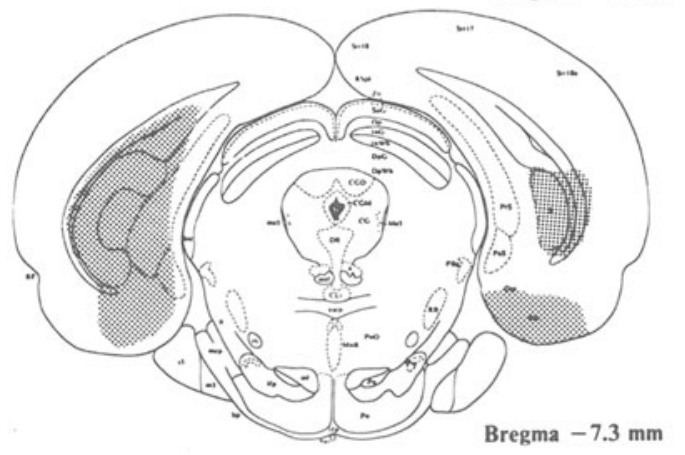

Hippocampal

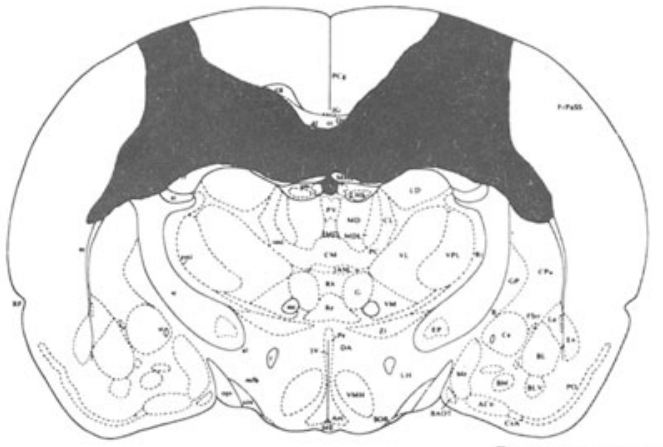

Bregma $-2.3 \mathrm{~mm}$

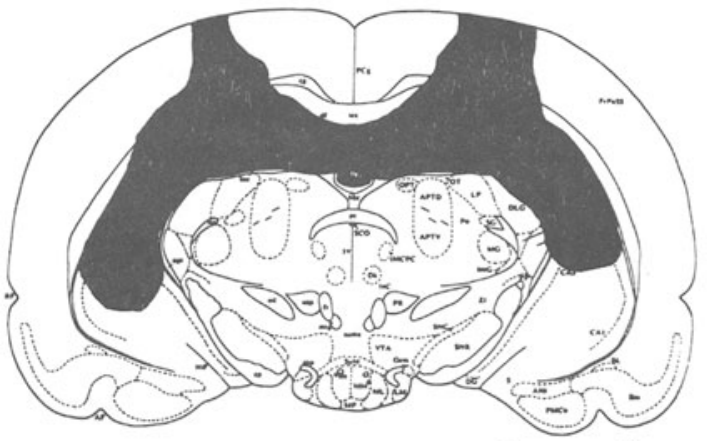

Bregma $-4.8 \mathrm{~mm}$

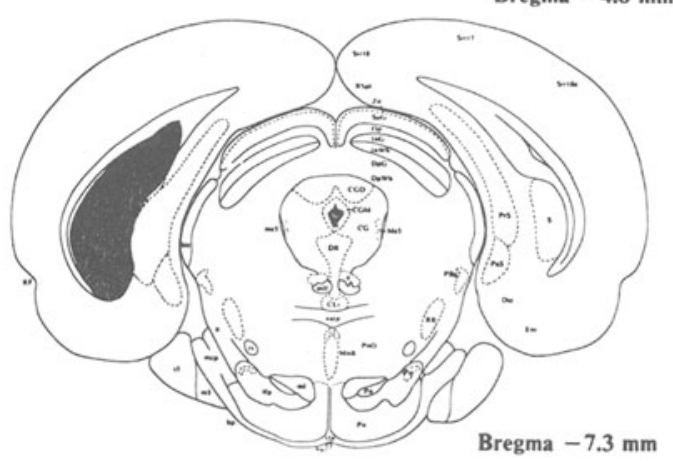

Amygdalar

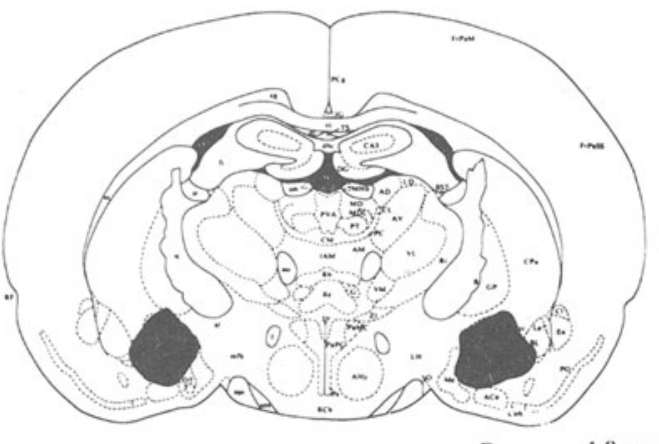

Bregma $-1.8 \mathrm{~mm}$

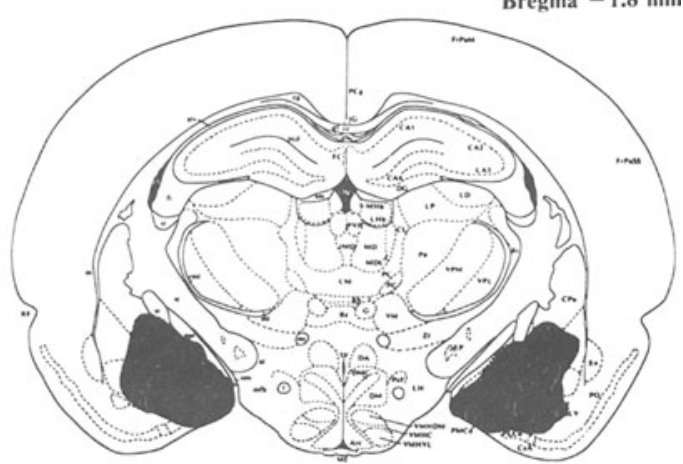

Bregma $-3.3 \mathrm{~mm}$

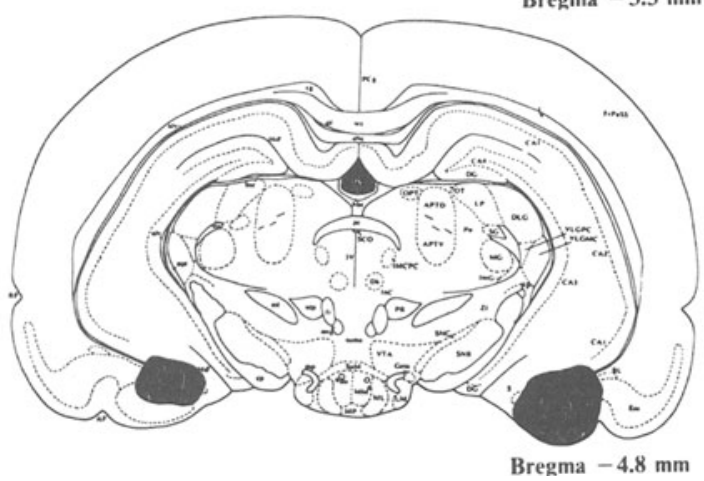

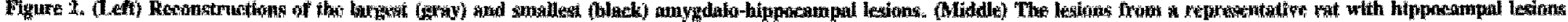

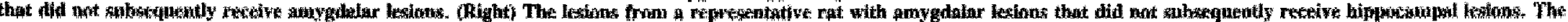

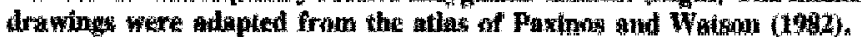



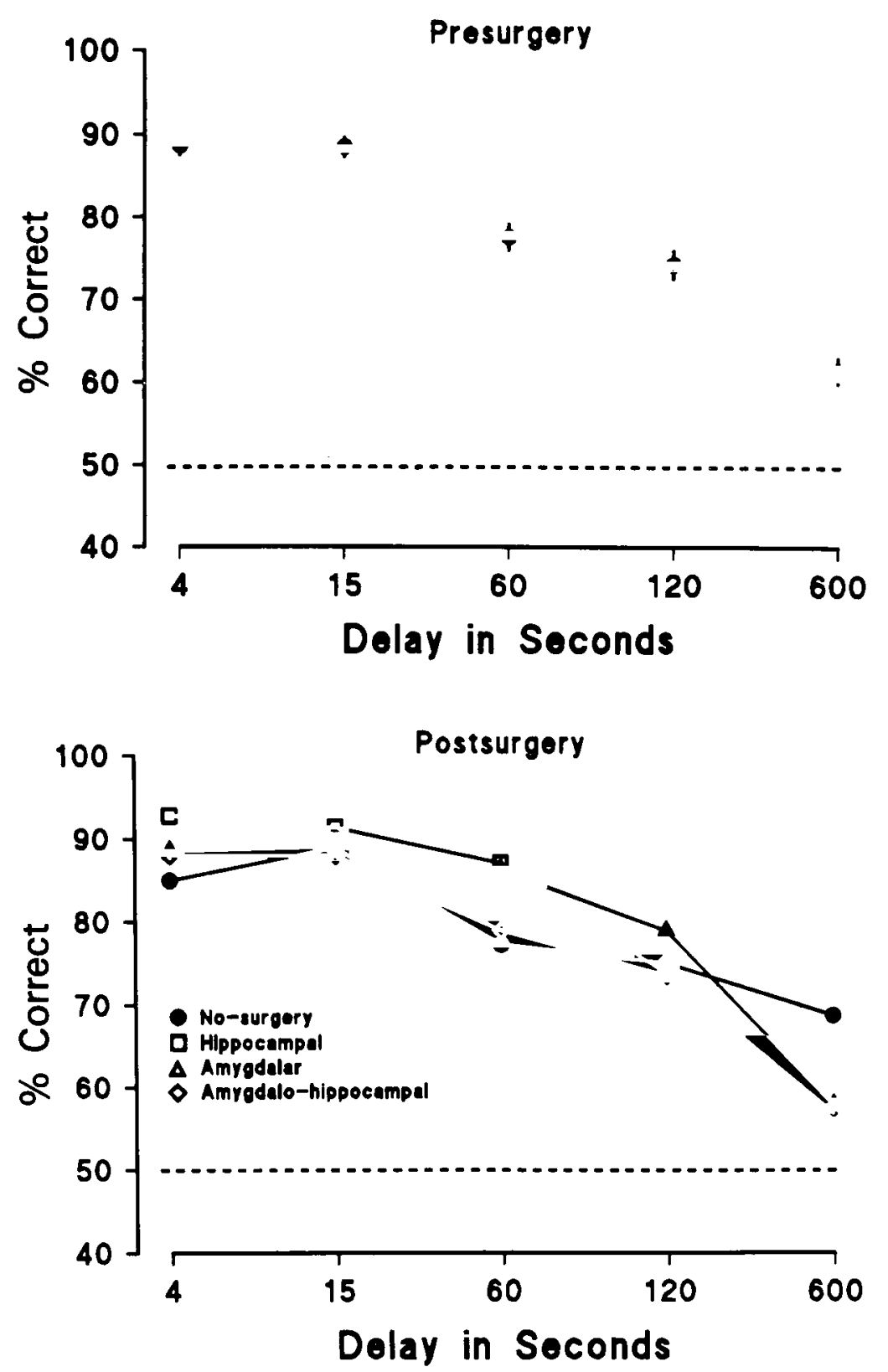

Figure 3. The presurgery (top) and postsurgery (bottom) retention functions that were determined on mixed-delay sessions. SEMs for postsurgery scores ranged from 0.97 to 3.70 .

Postsurgery testing: Retention functions. The mean postsurgery scores for each group on the mixed-delay sessions are illustrated in Figure 3. There were no statistically significant differences between the pre- and postsurgery scores of any of the groups at any delay. For multiple planned comparisons between the groups' postsurgery scores at each delay, we adopted a critical confidence level of .01 . Relative to the performance of the nosurgery control rats, postsurgery scores were significantly lower in all three experimental groups at the 600-sec de- lay $[t(11)=3.16$, for hippocampal lesions; $t(11)=3.34$, for amygdalar lesions; $t(20)=3.20$, for amygdalohippocampal lesions; all $p s<.01$ ]. These differences $\alpha$ curred because the scores in each experimental group at the 600-sec delay were slightly lower after surgery than before surgery, whereas the scores in the no-surgery control group were slightly higher after surgery than before surgery. One-sample $t$ tests revealed that the postsurgery scores at the 600-sec delay in each of the three experimental groups were still significantly better than chance (all 

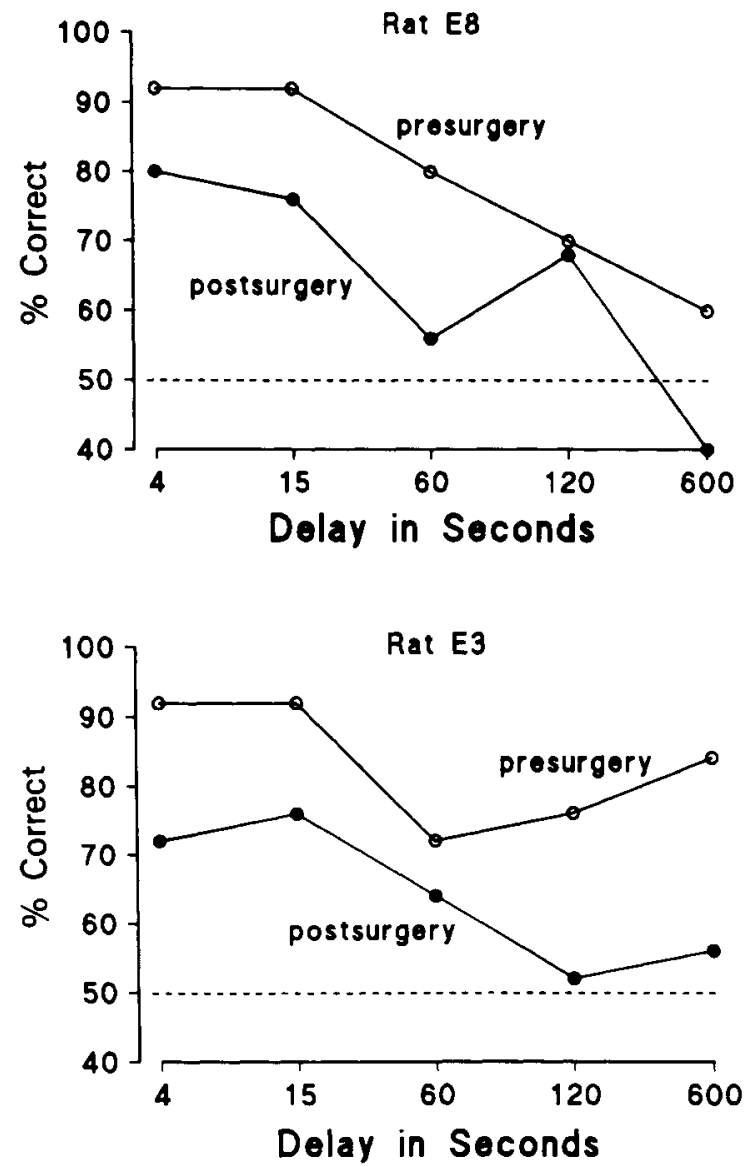

Figure 4. Presurgery (filled circles) and postsurgery (open circles) retention functions for two rats $(\mathrm{E} 3$ \& $\mathrm{E8})$ that sustained inadvertent damage to entorhinal, perirhinal, and temporal association cortex.

ps > .05; one-tailed). There were no statistically significant differences among the four groups at any of the delays shorter than $600 \mathrm{sec}$.

The 4 rats with hippocampal lesions that also sustained unintended damage to temporal association, perirhinal, and lateral entorhinal cortex displayed profound postsurgery impairments. One of them (E3) averaged $80 \%$ correct over the last 5 postsurgery sessions at the 4-sec delay (i.e., Sessions 15-20) but could not achieve the criterion; another one (E8) required 120 postsurgery sessions to reach the criterion, which was more than was required by any of the hippocampal-lesioned rats without damage to these areas of cortex. The pre- and postsurgery retention functions for these 2 rats are illustrated in Figure 4 , and reconstructions of their lesions are shown in Figure 5. Both of them displayed postsurgery deficits at all delays. The other 2 rats with unintended damage to entorhinal, perirhinal, and temporal association cortex averaged less than $65 \%$ correct over the last 5 postsurgery sessions at the 4-sec delay and thus were not subsequently tested on mixed-delay sessions.
The present experiment did not include a control group with lesions limited to that area of parietal neocortex that is removed during hippocampal aspiration, but we present data here from 2 rats with such lesions that were part of an earlier pilot experiment. Training and testing procedures in the pilot experiment were identical to those of the present experiment, except that the longest delay was $300 \mathrm{sec}$ instead of $600 \mathrm{sec}$. The mean presurgery scores of these 2 rats with posterior parietal cortex lesions were $95 \%, 85 \%, 75 \%, 78 \%$, and $78 \%$, at delays of $4,15,60$, 120 , and $300 \mathrm{sec}$, respectively; their mean postsurgery scores were $92 \%, 85 \%, 80 \%, 82 \%$, and $75 \%$.

\section{DISCUSSION}

All rats in this experiment received extensive presurgery training on the DNMS task. Then, the experimental rats received lesions of either the hippocampus, the amygdala, or both. All three groups displayed only mild postsurgery DNMS deficits; they were significantly impaired at delays of $600 \mathrm{sec}$ but not at delays of $120 \mathrm{sec}$ or less. These findings cast doubt on the idea that either the hippocampus or the amygdala is critically involved in object-recognition memory in rats.

Our observation of mild DNMS deficits following bilateral lesions of the hippocampus alone or the amygdala alone is consistent with similar findings in monkeys (Mishkin, 1978; Murray \& Mishkin, 1984) and with reported failures to observe DNMS impairments in rats with hippocampal lesions at delays of $30 \mathrm{sec}$ (Rothblat \& Kromer, 1991) and in rats with hippocampal or amygdalar lesions at delays of $60 \mathrm{sec}$ (Aggleton et al., 1986). Recently, Kesner (1991) reported that rats with hippocampal lesions were unimpaired on a DNMS task that was modeled after the one used in the present experiment. However, our observation of mild DNMS deficits following combined amygdalo-hippocampal lesions is inconsistent with usual reports. Profound DNMS impairments have been reported following amygdalo-hippocampal lesions both in monkeys (e.g., Mishkin, 1978; Zola-Morgan \& Squire, 1985) and in rats (Aggleton et al., 1989).

The results of recent studies with monkeys suggest one way of reconciling the present results with the numerous reports of DNMS impairments following combined lesions of the hippocampus and amygdala. They suggest that amygdalo-hippocampal lesions do not produce profound impairments on DNMS unless there is collateral damage to adjacent cortex. In primates, amygdalo-hippocampal lesions are usually created by aspiration and thus include damage to portions of the parahippocampal gyrus, entorhinal cortex, and, in some cases, perirhinal cortex (Murray \& Mishkin, 1986). Impairments on DNMS have been found in monkeys following lesions restricted to these cortical areas (Meunier, Murray, Bachevalier, \& Mishkin, 1990; Murray, Bachevalier, \& Mishkin, 1989; Zola-Morgan, Squire, Amaral, \& Suzuki, 1989). Furthermore, monkeys with combined amygdalo-hippocampal lesions that spare the cortex surrounding the amygdala (i.e., 


\section{Rat E8}

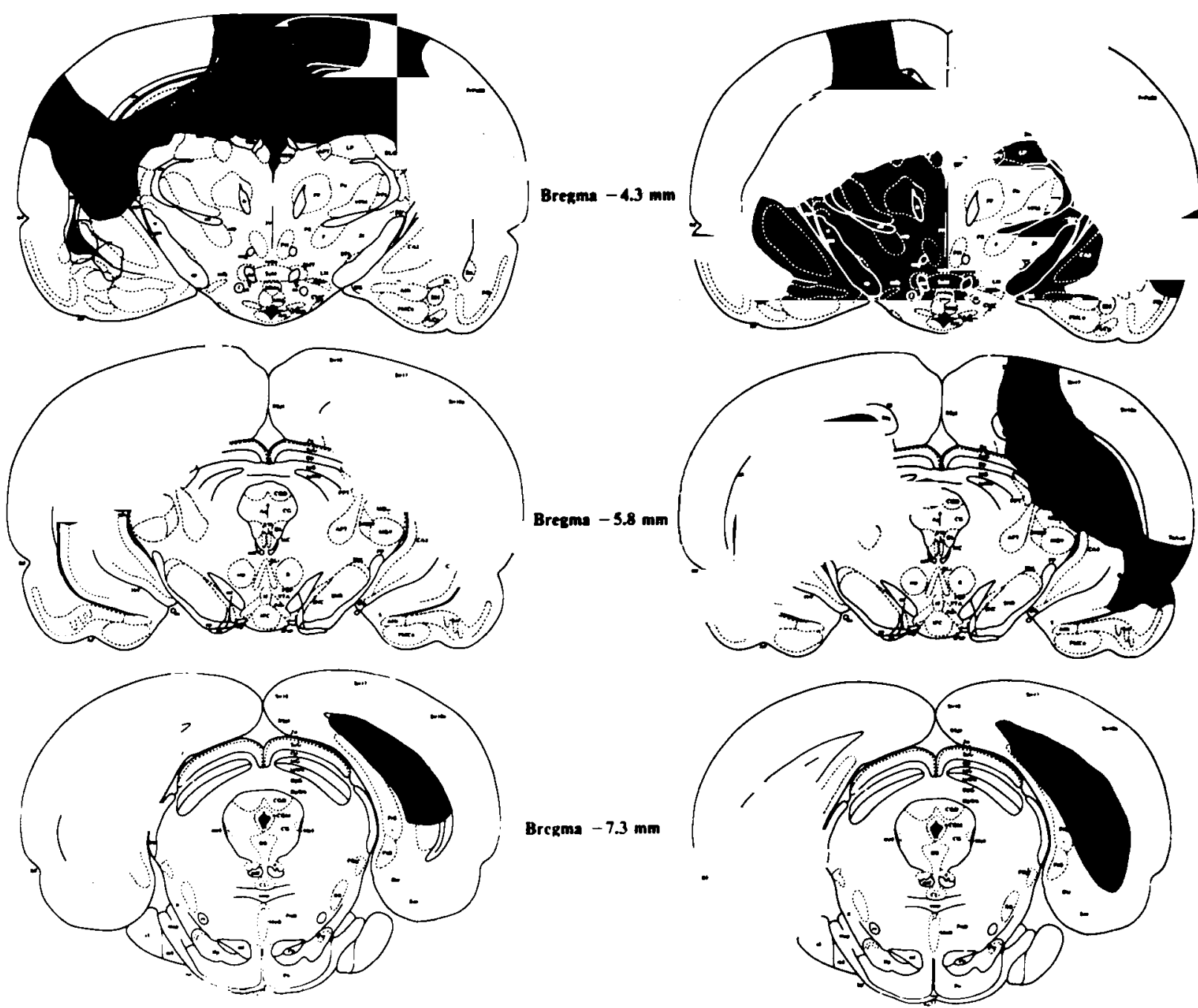

Figure 5. Lesions in two rats with hippocampal lesions (E3 \& E8) that sustained inadvertent damage to entorhinal, perirhinal, and temporal association cortex, and that were subsequently tested on mixed-delay sessions.

entorhinal, perirhinal, and periamygdaloid cortices) perform no worse on DNMS than do monkeys with lesions of the hippocampus and the parahippocampal cortex (ZolaMorgan, Squire, \& Amaral, 1989). This raises the question of whether the rats with amygdalo-hippocampal lesions in the present experiment were only mildly impaired on DNMS because associated cortical areas were spared. Indeed, the dorsal approach that we used to aspirate the hippocampus in rats caused damage primarily to posterior parietal association cortex (Kolb, 1990), and it spared entorhinal and perirhinal cortex.

In the present experiment, 4 of the rats with hippocampal lesions sustained unintended damage to various amounts of entorhinal and perirhinal cortex. They were the only subjects displaying a severe postsurgery DNMS impairment. It is possible that the damage to entorhinal and perirhinal cortex was responsible for their impairments, but it should be noted that each of them also sustained some damage to other structures that were not damaged in most of the rats with hippocampal lesions. Most notably, all 4 of these rats received either unilateral or bilateral damage to the lateral geniculate nucleus that was substantial, albeit incomplete, and it is possible that they suffered some degree of visual impairment as a consequence. Another possibilility is that their DNMS deficit was secondary to a visual discrimination deficit produced by damage to temporal association cortex. In monkeys, lesions in the homologous brain region-the inferotemporal cortex - produce impairments on visual discrimination tasks and impairments across all delays of a delayed matching-to-sample task (Horel, Pytko-Joiner, Voytko, $\&$ Salsbury, 1987). In the present experiment, the 4 rats 
with perirhinal and lateral entorhinal cortex lesions also sustained damage to temporal association cortex; 2 of them were tested on mixed-delay sessions, and both of them displayed postsurgery deficits across all delays. The possibility cannot be ruled out that the postsurgery DNMS impairments in these 4 rats were due to perceptual rather than memory deficits or to damage outside of perirhinal and entorhinal cortex. As a corollary, the hippocampal damage that they sustained was likely unessential in the production of their severe impairments because it was no more extensive than the damage sustained by the other rats with hippocampal lesions that displayed only mild impairments. Alternatively, it might have been a combination of the hippocampal lesions and some other damage that produced their impairments-perhaps neither alone would have done so.

There is a second factor-in addition to the lack of collateral damage to critical areas of cortex - that could have contributed to the good DNMS performance of the present subjects with amygdalo-hippocampal lesions. This factor is the extensive presurgery training that they received. Studies with monkeys suggest that the impairments on DNMS following hippocampal lesions are often greater in subjects that receive no presurgery training (e.g., Bachevalier \& Mishkin, 1989; Mahut et al., 1982; ZolaMorgan \& Squire, 1986) than in subjects that do (e.g., Bachevalier et al., 1985; Mishkin, 1978; Murray, 1990; Murray \& Mishkin, 1986). In Rothblat and Kromer's (1991) recent study, hippocampal lesions did not impair DNMS performance in rats that had received presurgery training. It is possible that the extensive presurgery training in the present experiment $(M=1,211$ presurgery trials; range $=825-1,500$ trials) made the rats' DNMS performance relatively insensitive to large hippocampal lesions. However, such an explanation cannot explain why Aggleton et al. (1986) found no impairment on DNMS in rats with hippocampal lesions that did not receive presurgery training. It is possible that amygdalar, hippocampal, or amygdalo-hippocampal lesions would cause impairments in rats with no presurgery training in the DNMS paradigm that we used in this study; however, such an experiment has not yet been conducted.

Although the present findings suggest that lesions limited to the hippocampus and amygdala do not cause severe impairments on DNMS, the statistically significant impairments at the 600-sec delay suggest that DNMS performance can be sensitive to such lesions if the task is made sufficiently difficult. In support of this notion, other studies have found that mild DNMS impairments in monkeys with hippocampal or amygdalar lesions can be accentuated by requiring them to remember lists of several sample items at one time (e.g., Mahut et al., 1982; Mishkin, 1978; Murray \& Mishkin, 1986). Raffaele and Olton (1988) found impairments on DNMS in rats with hippocampal lesions when only two stimuli were used throughout testing, thus increasing the degree of proactive interference over that which is present when different stimuli are used on each trial. Using a matching-to-sample procedure in which only two test stimuli were used throughout testing, Jagielo, Nonneman, Isaac, and Jackson-Smith (1990) found impaired performance in rats with hippocampal lesions.

Overall, the findings from this study strongly suggest that object-recognition memory in rats does not require an intact hippocampus or amygdala, at least not when it is assessed by DNMS performance in pretrained rats. There were some indications that DNMS performance is severely impaired in rats by damage to adjacent cortex, but this possibility requires systematic investigation. Generalizing from the present findings in rats to similar experiments with monkeys requires the assumption that rats and monkeys are using a similar strategy when solving the DNMS task, an assumption that cannot presently be confirmed. Even if this assumption is correct, differences in the anatomy and connectivity of limbic structures in rats and monkeys (see Witter, Groenewegen, Lopes de Silva, \& Lohman, 1989) might produce different functional consequences following limbic lesions in the two species.

\section{REFERENCES}

Aggleton, J. P., Blindt, H. S., \& Rawlins, J. N. P. (1989). Effects of amygdaloid and amygdaloid-hippocampal lesions on object recognition and spatial working memory in rats. Behavioral Neuroscience, 103, 962-974.

AgGleton, J. P., Hunt, P. R., \& Rawlins, J. N. P. (1986). The effects of hippocampal lesions upon spatial and non-spatial tests of working memory. Behavioral Brain Research, 19, 133-146.

Bachevalier, J., Mishkin, M. (1989). Mnemonic and neuropathological effects of occluding the posterior cerebral artery in Macaca mulatta. Neuropsychologia, 27, 83-105.

Bachevalier, J., Parkinson, J. K., \& Mishkin, M. (1985). Visual recognition in monkeys: Effects of separate vs. combined transsection of fornix and amygdalofugal pathways. Experimental Brain Research, 57, 554-561.

Horel, J. A., Pytko-Joiner, D. E., Voytko, M. L., \& Salsbury, K. (1987). The performance of visual tasks while segments of the inferior temporal cortex are suppressed by cold. Behavioral Brain Research, 23, 29-42.

JaGielo, J. A., Nonneman, A. J., ISAac, W. L., Jackson-Smith, P. A. (1990). Hippocampal lesions impair rats' performance of a nonspatial matching-to-sample task. Psychobiology, 18, 55-62.

KESNER, R. P. (1991). The role of the hippocampus within an attribute model of memory. Hippocampus, 1, 279-282.

KoLB, B. (1990). Posterior parietal and temporal association cortex. In B. Kolb \& R. C. Tess (Eds.), The cerebral cortex of the rat (pp. 459-471). Cambridge, MA: MIT Press.

MahUt, H., Zola-Morgan, S., \& Moss, M. (1982). Hippocampal resections impair associative learning and recognition memory in the monkey. Journal of Neuroscience, 9, 1214-1229.

Meunier, M., Murray, E. A., Bachevalier, J., Mishisin, M. (1990). Effects of perirhinal cortical lesions on visual recognition memory in rhesus monkeys. Society for Neuroscience Abstracts, 16, 616 .

Mishkin, M. (1978). Memory in monkeys severely impaired by combined but not by separate removal of amygdala and hippocampus. $\mathrm{Na}$ ture, 273, 297-298.

Mishkin, M., A APEnzeller, T. (1987). The anatomy of memory. Scientific American, 256, 62-71.

Mumby, D. G., Pinel, J. P. J., Woon, E. R. (1990). Nonrecurringitems delayed nonmatching-to-sample in rats: A new paradigm for testing nonspatial working memory. Psychobiology, 18, 321-326. 
MurRAY, E. A. (1990). Representational memory in nonhuman primates. In R. P. Kesner \& D. S. Olton (Eds.), Neurobiology of comparative cognition (pp. 127-155). Hillsdale, NJ: Erlbaum.

Murray, E. A., Bachevalier, J., Mishioin, M. (1989). Effects of rhinal cortical lesions on visual recognition memory in rhesus monkeys. Society for Neuroscience Abstracts, 15, 342.

Murray, E. A., Mishkin, M. (1984). Severe tactual as well as visual memory deficits follow combined removal of the amygdala and hippocampus in monkeys. Journal of Neuroscience, 4, 2565-2580.

MuRRAY, E. A., MishKIN, M. (1986). Visual recognition in monkeys following thinal cortical ablations combined with either amygdalectomy or hippocampectomy. Journal of Neuroscience, 6, 1991-2003.

Olton, D. S., * Feustle, W. A. (1981). Hippocampal function required for nonspatial working memory. Experimental Brain Research, 41, 380-389.

PAxinos, G., W WATSON, C. (1982). The rat brain in stereotaxic coordinates. Toronto: Academic Press.

RAFFAele, K. C., Olton, D. S. (1988). Hippocampal and amygdaloid involvement in working memory for non-spatial stimuli. Behavioral Neuroscience, 102, 349-355.

Rothblat, L. A., \& Kromer, L. F. (1991). Object recognition memory in the rat: The role of the hippocampus. Behavioural Brain Research, 42, 25-32.

SQuire, L. R., Zola-Morgan, S., \& Chen, K. S. (1988). Human amnesia and animal models of amnesia: Performance of amnesic patients on tests designed for the monkey. Behavioral Neuroscience, 102, 210-221.

Witter, M. P., Groenewegen, H. J., Lopes de Silva, F. H., * LohmaN, A. H. M. (1989). Functional organization of the extrinsic and intrinsic circuitry of the parahippocampal region. Progress in Neurobiology, 33, 161-253.

ZilLes, K. (1985). The cortex of the rat: A stereotaxic atlas. Berlin: Springer-Verlag.

Zola-Morgan, S., Squire, L. R. (1985). Medial temporal lesions in monkeys impair memory on a variety of tasks sensitive to human amnesia. Behavioral Neuroscience, 99, 22-34.

Zola-Morgan, S., \& SQuire, L. R. (1986). Memory impairment in monkeys following lesions of the hippocampus. Behavioral Neuroscience, 100, 165-170.

Zola-Morgan, S., Squire, L. R., Amaral, D. G. (1989). Lesions of the amygdala that spare adjacent cortical regions do not impair memory or exacerbate the impairment following lesions of the hippocampal formation. Joumal of Neuroscience, 9, 1922-1936.

Zola-Morgan, S., SQuire, L. R., Amaral, D. G., Suzuki, W. A. (1989). Lesions of perirhinal and parahippocampal cortex that spare the amygdala and hippocampal formation produce severe memory impairment. Journal of Neuroscience, 9, 4355-4370.

(Manuscript received August 27, 1991; revision accepted for publication December 5, 1991.) 